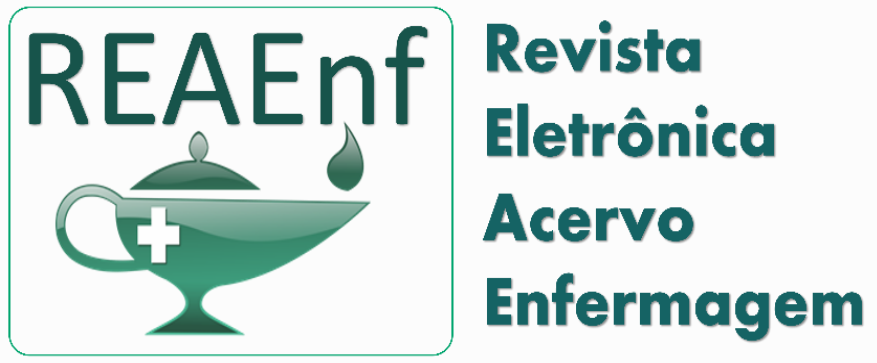

\section{ARTIGO ORIGINAL}

Recebido em: 11/2020

Aceito em: $12 / 2020$

Publicado em: 1/2021

\title{
Perfil de pacientes com câncer de próstata atendidos em um centro de oncologia
}

\author{
Profile of patients with prostate cancer care at an oncology center
}

Perfil de pacientes con atención de cáncer de próstata en un centro de oncología

Mariana Gonçalves Andrade ${ }^{1}$, Iris Edná Pereira da Silva ${ }^{2 *}$, Dálete de Souza Coelho Silva², Virgínia lone Araújo de Souza ${ }^{2}$, Faraildes Maria Cavalcanti Almeida ${ }^{3}$, Lidiane Régia Pereira Braga de Britto'.

Resumo: O câncer de próstata é a neoplasia maligna visceral mais comum no homem com exceção dos tumores cutâneos, cuja incidência tende a aumentar nas próximas décadas devido ao progresso da expectativa de vida. Embora os fatores de risco para sua ocorrência não estejam totalmente elucidados, a idade avançada, etnia e predisposição familiar representam fatores determinantes. O presente trabalho consiste em identificar o perfil dos pacientes acometidos com câncer de próstata atendidos em um centro de referência em oncologia caracterizando-se por um estudo descritivo. Foi aplicado um questionário estruturado em 88 pacientes diagnosticados com câncer de próstata e analisados os prontuários dos mesmos. Pode-se constatar que houve uma prevalência de homens com idade entre 71 e 80 anos $(47,72 \%)$, analfabetos $(38,63 \%)$, casados $(73,86 \%)$, agricultores $(56,81 \%)$ e procedentes de cidades com até 20 mil habitantes. Com relação às variáveis referentes aos fatores de risco, evidenciou o predomínio de homens com IMC normal (47,72\%), que não seguem uma dieta alimentar restritiva $(70,45 \%)$ e no qual apresentam um alto consumo de gordura animal (52,27\%), carne vermelha $(69,31 \%)$ e leite e derivados $(63,63 \%)$. O conhecimento das características dos homens acometidos com câncer de próstata na região poderá nortear políticas públicas direcionadas para essa população.

Palavras-chave: Câncer de próstata, Epidemiologia, Fatores de risco.

\begin{abstract}
Prostate cancer is the most common visceral malignancy in men with the exception of skin tumors, the incidence of which tends to increase in the coming decades due to the progress in life expectancy. Although the risk factors for its occurrence are not fully understood, advanced age, ethnicity and family predisposition represent determining factors. The present work consists of identifying the profile of patients affected with prostate cancer treated at a reference center in oncology, characterized by a descriptive study. A structured questionnaire was applied to 88 patients diagnosed with prostate cancer and their medical records were analyzed. It can be seen that there was a prevalence of men aged between 71 and 80 years $(47.72 \%)$, illiterate $(38.63 \%)$, married $(73.86 \%)$, farmers $(56.81 \%)$ and coming from cities with
\end{abstract}

\footnotetext{
1 Universidade de Pernambuco, Recife - PE.

2 Faculdade de Ciências Médicas, Recife - PE. *E-mail: iris.psilva@upe.br

${ }^{3}$ Faculdade Maurício de Nassau, Recife - PE.
} 
up to 20 thousand inhabitants. Regarding the variables related to risk factors, the predominance of men with normal BMI (47.72\%), who do not follow a restrictive diet $(70.45 \%)$ and in which they have a high consumption of animal fat (52), $27 \%$ ), red meat (69.31\%) and milk and dairy products (63.63\%). Knowledge of the characteristics of men affected with prostate cancer in the region may guide public policies aimed at this population.

Keywords: Prostate cancer, Epidemiology, Risk factors.

Resumen: El cáncer de próstata es la neoplasia visceral más común en los hombres, a excepción de los tumores de piel, cuya incidencia tiende a aumentar en las próximas décadas debido al avance de la esperanza de vida. Aunque los factores de riesgo para su aparición no se comprenden completamente, la edad avanzada, la etnia y la predisposición familiar representan factores determinantes. El presente trabajo consiste en identificar el perfil de pacientes afectos de cáncer de próstata atendidos en un centro de referencia en oncología, caracterizado por un estudio descriptivo. Se aplicó un cuestionario estructurado a 88 pacientes diagnosticados de cáncer de próstata y se analizaron sus historias clínicas. Se puede observar que hubo una prevalencia de hombres entre 71 y 80 años $(47,72 \%)$, analfabetos $(38,63 \%)$, casados $(73,86 \%)$, agricultores $(56,81 \%)$ y provenientes de ciudades con hasta 20 mil habitantes. En cuanto a las variables relacionadas con los factores de riesgo, el predominio de hombres con IMC normal $(47,72 \%)$, que no siguen una dieta restrictiva $(70,45 \%)$ y en los que tienen un alto consumo de grasa animal (52), $27 \%$ ), carnes rojas $(69,31 \%)$ y leche y productos lácteos $(63,63 \%)$. El conocimiento de las características de los hombres afectados por cáncer de próstata en la región puede orientar las políticas públicas dirigidas a esta población.

Palabras clave: Cáncer de próstata, Epidemiología, Factores de riesgo.

\section{INTRODUÇÃO}

O câncer de próstata é a neoplasia maligna visceral mais comum no homem excetuando se os tumores cutâneos e, a incidência tende a crescer nas próximas décadas com o aumento da expectativa de vida (NASSIF AE, et al., 2009). No Brasil, estimam-se a incidência de câncer de próstata de 65.840. Esses valores correspondem a um risco estimado de 62,92 casos novos a cada 100 mil homens. Sem considerar os tumores de pele não melanoma, o câncer de próstata foi o mais incidente entre os homens em todas as regiões do país, com 72,35/100 mil na Nordeste, 65,29/100 mil na Centro Oeste, 63,94/100 mil na Sudeste, 62,00/100 mil na Sul e 29,39/100 mil na Norte. Segundo o Sistema de Informação de Mortalidade em 2018, foram registrados 15.576 óbitos. Dos estados da região nordeste, Pernambuco ocupa $\circ 3^{\circ}$ lugar com estimativa de 2.630 casos novos, desses 590 são da cidade do Recife (INCA, 2020).

Os principais fatores de risco descritos para o desenvolvimento do câncer de próstata são: idade avançada, etnia e predisposição familiar (CZORNY RCN, et al., 2017). Hábitos alimentares estão intimamente ligados ao risco de desenvolvimento da neoplasia prostática, tais como o alto consumo energético, ingestão de carne vermelha, gorduras, leite e álcool (PAIVA EP, et al., 2010).

Vários aspectos favorecem o crescimento da doença no país, como a melhoria e a evolução dos métodos diagnósticos, a melhoria da qualidade dos sistemas de informação e a ocorrência de sobre diagnóstico (INCA, 2020). Devido às consequências de grande impacto sobre a população masculina no que diz respeito à morbimortalidade, o câncer de próstata representa um problema de saúde pública. Além disso, deve ser considerada por questões socioeconômicas, visto que, com o envelhecimento populacional e consequente aumento da expectativa de vida, a doença tende a ser mais presente (DINI LI e KOFF WJ, 2006).

As influências socioculturais, incluindo os estereótipos de gênero, crenças e valores que definem o que é ser masculino, têm sido apontados como obstáculos na implementação de práticas de cuidado em saúde neste grupo populacional. Neste contexto, o câncer de próstata torna-se um tema muito difícil de ser discutido, limitando suas possibilidades de cuidado (MOSCHETA MS e SANTOS MA, 2012).

REAEnf/EJNC | Vol. 8 | e5855 | DOI: https://doi.org/10.25248/REAenf.e5855.2021 Página 2 de 9 
A caracterização de homens acometidos com câncer de próstata, mesmo que de forma regional é relevante, pois informações sobre os dados epidemiológicos e socioculturais podem nortear ações na Atenção Básica, podendo assim assistir a essa população de maneira precoce e eficaz. Diante desse pressuposto, este estudo teve por objetivo identificar o perfil dos pacientes acometidos com câncer de próstata atendidos no centro de referência em oncologia.

\section{MÉTODOS}

Trata-se de um estudo de natureza descritiva, tendo como participantes da pesquisa 88 pacientes diagnosticados com câncer de próstata, onde foram eleitos por conveniência. Como critérios de inclusão foram utilizados: pacientes diagnosticados com câncer de próstata e pacientes com câncer de próstata em processo metastático. A pesquisa foi realizada em um centro de oncologia atendidos em uma cidade de Pernambuco, no período de março de 2016 a junho de 2017.

Para a coleta de dados foi utilizado um questionário estruturado para obter dados referentes às características socioeconômicas, história clínica e hábitos alimentares e comportamentais dos pesquisados. Além disso, foi utilizado um formulário, de autoria do pesquisador, onde foi possível analisar os prontuários e colher informações sobre o Escore de Gleason ao diagnóstico, classificação da doença, sintomas ao tratamento e revisão por Antígeno Prostático Específico (PSA) e toque retal.

Após a aplicação dos questionários e análise dos prontuários, as informações foram transcritas para um banco de dados a partir de uma planilha no programa Microsoft Office Excel 2016. Em seguida, houve a tabulação com uma análise descritiva dos dados das mesmas e a distribuição das frequências absolutas e relativas.

Todos os participantes da pesquisa assinaram o Termo de Consentimento Livre e Esclarecido (TCLE) de acordo com a resolução $n^{\circ} 466 / 12$ do Conselho Nacional de Saúde (CNS) e o projeto foi aprovado pelo Comitê de Ética e Pesquisa (CEP)/UPE, sob número do parecer: 2.156.490 (CAAE 59223316.5.0000.5207).

\section{RESULTADOS}

Foram entrevistados e analisados os prontuários de 88 pacientes. Dentre eles, prevaleceram homens com idade entre 71 e 80 anos $(47,72 \%)$, analfabetos (38,63\%), casados $(73,86 \%)$, agricultores $(56,81 \%)$ e procedentes de cidades com até 20 mil habitantes, mostrados na (Tabela 1).

Dentre outras profissões exercidas foram encontradas: funcionário público, mestre de obras, mecânico e vigilante, contando cada um com $2,27 \%$ do percentual da amostra. Além destes, verificou-se a ocorrência de outras ocupações como pecuarista, cabelereiro, contador, açougueiro, operador de máquinas, guarda florestal, eletricista, pescador, autônomo e pedreiro, cada um correspondendo a 1,14\% da amostra do estudo. Acerca da procedência, verificou-se que 57,95\% são do estado Pernambuco, 32,95\% são da Bahia, $5,68 \%$ são do Piauí e $1,14 \%$ são do Ceará. 
Tabela 1 - Variáveis sociodemográficas dos pacientes acometidos com câncer de próstata atendidos em uma cidade de Pernambuco.

\begin{tabular}{|c|c|c|}
\hline Variáveis sociodemográficas & $\mathbf{N}$ & $\%$ \\
\hline \multicolumn{3}{|l|}{ Faixa etária ao diagnóstico } \\
\hline 40 a 50 anos & 2 & 2,27 \\
\hline 51 a 60 anos & 2 & 2,27 \\
\hline 61 a 70 anos & 13 & 14,77 \\
\hline 71 a 80 anos & 42 & 47,72 \\
\hline 81 a 90 anos & 25 & 28,4 \\
\hline 90 anos ou mais & 4 & 4,54 \\
\hline \multicolumn{3}{|l|}{ Escolaridade } \\
\hline Analfabeto & 34 & 38,63 \\
\hline Ensino fundamental incompleto & 32 & 36,36 \\
\hline Ensino fundamental completo & 15 & 17,04 \\
\hline Ensino médio incompleto & 1 & 1,14 \\
\hline Ensino médio completo & 6 & 6,81 \\
\hline \multicolumn{3}{|l|}{ Estado Conjugal } \\
\hline Casado & 65 & 73,86 \\
\hline Viúvo & 17 & 19,32 \\
\hline Solteiro & 2 & 2,27 \\
\hline Divorciado & 4 & 4,54 \\
\hline \multicolumn{3}{|l|}{ Profissão } \\
\hline Agricultor & 50 & 56,81 \\
\hline Lavrador & 9 & 10,22 \\
\hline Motorista & 4 & 4,54 \\
\hline Comerciante & 3 & 3,4 \\
\hline Policial & 3 & 3,4 \\
\hline Não informado & 1 & 1,14 \\
\hline \multicolumn{3}{|c|}{ Densidade demográfica da cidade de procedência } \\
\hline Até $20 \mathrm{mil}$ & 27 & 30,68 \\
\hline 21 a 50 mil & 21 & 23,86 \\
\hline 51 a $100 \mathrm{mil}$ & 17 & 19,32 \\
\hline Mais de $100 \mathrm{mil}$ & 22 & 25 \\
\hline Não informado & 1 & 1,14 \\
\hline
\end{tabular}

Fonte: Andrade MG, et al., 2020.

Observam-se que na Tabela 2 há as informações alimentares e comportamentais, onde se pode evidenciar o predomínio de homens com IMC normal (47,72\%), que não seguem dieta alimentar restritiva $(70,45 \%)$ e no qual apresentam um alto consumo de gordura animal $(52,27 \%)$, carne vermelha $(69,31 \%)$ e de leite e derivados $(63,63 \%)$.

Com relação ao tabagismo, $92,04 \%$ dos homens não eram fumantes, sendo que, $60,22 \%$ dessa amostra apresentavam uma história pregressa de uso do tabaco. Dessa forma, levando em consideração o tempo de abstinência, houve uma prevalência de $69,81 \%$ de pacientes que possuíam mais de 10 anos sem o uso do tabaco, seguido de 22,64\% que possuíam entre 1 e 5 anos e $7,55 \%$ que possuíam entre 6 e 10 anos sem fumar. Além disso, $84,09 \%$ dos pacientes não tinham convívio com fumantes.

De acordo com a distribuição de pacientes que consomem bebidas alcoólicas, 92,04\% não apresentam esse hábito, porém a maioria dos participantes da pesquisa possuía uma história pregressa de ingesta de bebidas alcoólicas durante a vida. Além disso, 46,59\% dos homens faziam uso ocupacional de agrotóxicos, sendo que $53,65 \%$ tinham contato semanalmente com esses produtos, $19,51 \%$ mensalmente, $17,07 \%$ anualmente, $4,87 \%$ a cada seis meses, $2,43 \%$ a cada três meses e $2,43 \%$ não soube informar a frequência do uso. 
Tabela 2 - Características alimentares e comportamentais dos pacientes acometidos com câncer de próstata atendidos em uma cidade de Pernambuco.

\begin{tabular}{|c|c|c|}
\hline Informações alimentares e comportamentais & $\mathbf{N}$ & $\%$ \\
\hline Abaixo do peso & 12 & 13,63 \\
\hline Acima do peso & 13 & 14,77 \\
\hline Marginalmente acima do peso & 8 & 9,09 \\
\hline Normal & 42 & 47,72 \\
\hline Obeso & 6 & 6,81 \\
\hline Não informado & 7 & 7,95 \\
\hline \multicolumn{3}{|l|}{ Segue dieta alimentar restritiva } \\
\hline Sim & 26 & 29,54 \\
\hline Não & 62 & 70,45 \\
\hline \multicolumn{3}{|l|}{ Consumo de gordura animal na semana } \\
\hline Não consome & 22 & 25 \\
\hline $1 \mathrm{vez}$ & 7 & 7,95 \\
\hline 2 vezes & 12 & 13,63 \\
\hline 3 vezes & 46 & 52,27 \\
\hline Não informado & 1 & 1,14 \\
\hline \multicolumn{3}{|l|}{ Consumo de carne vermelha na semana } \\
\hline Não consome & 4 & 4,54 \\
\hline $1 \mathrm{vez}$ & 5 & 5,68 \\
\hline 2 vezes & 17 & 19,31 \\
\hline 3 vezes & 61 & 69,31 \\
\hline Não informado & 1 & 1,13 \\
\hline \multicolumn{3}{|l|}{ Consumo de leite e derivados na semana } \\
\hline Não consome & 14 & 15,9 \\
\hline $1 \mathrm{vez}$ & 8 & 9 \\
\hline 2 vezes & 9 & 10,22 \\
\hline 3 vezes & 56 & 63,63 \\
\hline Não informado & 1 & 1,13 \\
\hline \multicolumn{3}{|l|}{ Prática de atividade física na semana } \\
\hline Não pratica & 57 & 64,77 \\
\hline 2 vezes & 7 & 22,58 \\
\hline 3 vezes & 23 & 74,19 \\
\hline Não informado & 1 & 3,22 \\
\hline \multicolumn{3}{|l|}{ Tabagismo } \\
\hline Fumantes & 7 & 7,95 \\
\hline Não fumantes & 81 & 92,04 \\
\hline Ex-fumantes & 53 & 60,22 \\
\hline Convívio com fumantes & 14 & 15,90 \\
\hline \multicolumn{3}{|l|}{ História familiar de câncer de próstata $(n=83)$} \\
\hline Parente de $1^{\circ}$ grau & 16 & 18,18 \\
\hline Parente de $2^{\circ}$ grau & 18 & 20,45 \\
\hline Parente de $3^{\circ}$ grau & 5 & 5,68 \\
\hline Parente de $4^{\circ}$ grau & 2 & 2,27 \\
\hline Não possui & 42 & 47,72 \\
\hline Não informado & 5 & 5,68 \\
\hline Mais de um parente acometido & 10 & 11,36 \\
\hline \multicolumn{3}{|l|}{ Contato com agrotóxico no trabalho $(\mathrm{n}=88)$} \\
\hline Sim & 41 & 46,59 \\
\hline Não & 47 & 53,4 \\
\hline
\end{tabular}

Fonte: Andrade MG, et al., 2020.

No que diz respeito à história familiar de câncer, 51,14\% possuíam parentes acometidos com essa patologia, destes $15,90 \%$ são parentes de $2^{\circ}$ grau. Já com relação à história familiar de câncer de próstata, $52,28 \%$ apresentaram familiares acometidos com a doença, tendo um maior percentual os parentes de $2^{\circ}$ grau $(20,45 \%)$. Verificou-se que $60,22 \%$ dos pacientes eram hipertensos, $19,32 \%$ eram diabéticos e 29,54\% eram acometidos com as duas doenças crônicas não transmissíveis. 
A Tabela 3 consta as variáveis clínicas encontradas no estudo, onde o estadiamento predominante foi 0 Gleason 7 (22,73\%), seguido do Gleason 6 (21,59\%). A classificação T4 da patologia esteve presente em $25 \%$ dos casos, porém em $31,81 \%$ dos prontuários não continham essa informação. Vale ressaltar que as classificações T1b N0 M0, T1b NX MX, T2, T2 N0 M0, T2 N1 M1, T2 NX M0, T2 NX MX, T2b N0 MX, T2c N1 MX, T2c MX N1, T3 NX M0, T3 NX MX, T3c, T4 M1, T4 N0 M1, T4 N1 M1b representaram 1,14\% cada do total da amostra.

De acordo com a sintomatologia durante o tratamento foi constatado que $36,36 \%$ dos casos não apresentaram nenhum sintoma, porém houve uma frequência significativa de dor óssea $(22,72 \%)$ e nictúria $(11,36 \%)$. Anemia severa, constipação, diarreia, dificuldade em deambular, edema em membros inferiores, fogachos, hipoestesia em membros inferiores, lesão vesical, lombalgia severa e perda de peso representaram $1,14 \%$ cada do total dos sintomas encontrados. Além disso, observou-se que $95,45 \%$ dos pacientes acometidos com câncer de próstata não realizaram vasectomia.

Tabela 3 - Variáveis clínicas dos pacientes acometidos com câncer de próstata atendidos em um hospital de Pernambuco.

\begin{tabular}{|c|c|c|}
\hline Variáveis clínicas & $\mathbf{N}$ & $\%$ \\
\hline Gleason 2 & 1 & 1,14 \\
\hline Gleason 3 & 6 & 6,82 \\
\hline Gleason 4 & 6 & 6,82 \\
\hline Gleason 5 & 9 & 10,23 \\
\hline Gleason 6 & 19 & 21,59 \\
\hline Gleason 7 & 20 & 22,73 \\
\hline Gleason 8 & 12 & 13,64 \\
\hline Gleason 9 & 3 & 3,4 \\
\hline Gleason 10 & 2 & 2,27 \\
\hline Não consta no prontuário & 10 & 11,36 \\
\hline \multicolumn{3}{|l|}{ Classificação } \\
\hline $\mathrm{T} 4$ & 22 & 25 \\
\hline T4 NO MO & 5 & 5,68 \\
\hline T4 N1 M1 & 3 & 3,4 \\
\hline T4 NX M0 & 10 & 11,36 \\
\hline T4 NX MX & 4 & 4,54 \\
\hline Não consta no prontuário & 28 & 31,81 \\
\hline \multicolumn{3}{|l|}{ Sintomas ao tratamento } \\
\hline Dor muscular, incontinência urinaria, sincope & 2 & 2,27 \\
\hline Polaciúra, disfunção erétil, ginecomastia, obstrucão uretral, paresia em MMII & 3 & 3,4 \\
\hline Parestesia em MMII & 4 & 4,54 \\
\hline Artralgia, disúria & 5 & 5,68 \\
\hline Nictúria & 10 & 11,36 \\
\hline Dor óssea & 20 & 22,72 \\
\hline Assintomático & 32 & 36,36 \\
\hline \multicolumn{3}{|l|}{ Revisão por PSA } \\
\hline Mensalmente & 12 & 13,63 \\
\hline A cada 2 meses & 2 & 2,27 \\
\hline A cada 3 meses & 43 & 48,86 \\
\hline A cada 4 meses & 2 & 2,27 \\
\hline A cada 6 meses & 29 & 32,95 \\
\hline \multicolumn{3}{|l|}{ Revisão por Toque Retal } \\
\hline A cada 3 meses & 3 & 3,40 \\
\hline A cada 6 meses & 2 & 2,27 \\
\hline Anualmente & 2 & 2,27 \\
\hline Não realiza & 81 & 92.04 \\
\hline
\end{tabular}

Fonte: Andrade MG, et al., 2020. 


\section{DISCUSSÃO}

No presente estudo houve uma predominância de pacientes com idade entre 71 e 80 anos, seguidos de pacientes entre 61 e 70 anos acometidos com câncer de próstata, corroborando assim com a literatura, onde cerca de $65 \%$ dos casos são diagnosticados em pacientes com idade superior a 65 anos (ABAZA R et al., 2006). Em estudos de necropsias têm sido observado que o risco dessa patologia eleva-se progressivamente com o envelhecimento (HAAS GP, et al., 2008).

Verificou-se que homens analfabetos e com o ensino fundamental incompleto representaram aproximadamente $75 \%$ da amostra estudada. Em outro estudo foi possível observar que a maioria dos homens apresentaram um baixo nível de escolaridade apontando convergência nos dados desta pesquisa (GONÇALVES IR et al., 2008). Esse perfil deve ser reflexo do grau de instrução do homem sexagenário em geral, uma vez que, em $2019,51,2 \%$ da população brasileira não haviam completado o ensino médio fundamental ou eram analfabetos e apenas $16,5 \%$ tinham concluído o ensino superior (IBGE, 2019).

Nesta pesquisa, o estado conjugal reflete a realidade confirmada por outro estudo nacional que apresentou $77 \%$ dos homens casados (ZACCHI SR, et al., 2014). Pesquisas internacionais observaram que homens solteiros, divorciados e viúvos apresentaram um maior risco de morte em comparação aos homens casados (KRAVDAL A, 2002; ABDOLLAH F, et al., 2011). Como justificativa, os autores do estudo relataram que os homens não casados podem ter menos apoio para se submeterem a um tratamento curativo, um estilo de vida menos comedido e alterações imunológicas, pelo estado conjugal (ZACCHI SR, et al., 2014).

No que se refere ao estilo de vida, houve um predomínio de homens com IMC normal, porém a maioria não segue uma dieta alimentar e apresentam uma alta ingestão de gordura animal, carne vermelha e leite e derivados durante a semana. A influência que a dieta pode exercer sobre a gênese do câncer ainda é incerta. Porém, tem sido apontada uma relação positiva entre o alto consumo energético total e ingestão de carne vermelha, gorduras e leite e o risco de câncer da próstata (BRASIL, 2002).

Quanto ao tabagismo, 92,04\% dos homens não eram fumantes, sendo que 60,22\% destes eram exfumantes. Com relação ao consumo de bebidas alcoólicas, a maioria não apresentou esse hábito, porém tinham história pregressa de ingestão durante a vida. Verificou-se que a maioria dos pacientes não pratica atividade física e não fizeram vasectomia. A literatura não apresenta um consenso em relação a esses fatores de risco, pois, de acordo com Gronberg H (2003), o tabagismo, a realização de vasectomia, etilismo e sedentarismo foram excluídos como fatores de risco. Por outro lado, estudos do INCA afirmaram que esses fatores são passíveis de influenciar potencialmente o desenvolvimento do câncer de próstata (BRASIL, 2002).

Considerando a exposição a agrotóxicos e câncer de próstata cerca da metade dos pacientes tiveram contato com esse tipo de produto químico com frequência. Essas substâncias agem como disruptores endócrinos, ocasionando distúrbios relacionados à reprodução humana, infertilidade masculina, anormalidades do desenvolvimento sexual e o surgimento de tumores hormônios-dependentes, como os cânceres de ovário, mama, próstata, testículos e tireóide (KUMAR V, et al., 2010). Além disso, vale ressaltar que o local de realização do estudo, está situado em uma região onde a economia é baseada na agricultura e exportação, desse modo, a exposição aos agrotóxicos nessa localidade é mais intensa tanto de forma direta, quanto indireta.

Com relação à história familiar de câncer de próstata mais da metade dos pacientes possuíam parentes acometidos com essa patologia, sendo a maioria (20,45\%) parentes de $2^{\circ}$ grau. De acordo com a literatura, se um parente de primeiro grau tem a doença, o risco é no mínimo duas vezes maior do indivíduo ter câncer de próstata. Se dois ou mais indivíduos da mesma família são afetados, o risco aumenta em 5 a 11 vezes. Porém, a hereditariedade não tem grande relevância no prognóstico da doença ou na influência da mortalidade relacionada ao câncer de próstata (SIDDIQUI AS, et al., 2006).

$\mathrm{Na}$ atual casuística, a maior parte dos casos apresentavam Gleason 7 (22,73\%) e Gleason $6(21,59 \%)$, sendo que, houve um predomínio da classificação T4 da doença. Porém, em 31,81\% dos prontuários não

REAEnf/EJNC | Vol. 8 | e5855 | DOI: https://doi.org/10.25248/REAenf.e5855.2021 Página 7 de 9 
foi mencionado essa informação. À vista disso, estudos demonstraram que a maioria dos casos são descobertos quando os tumores estão restritos somente a próstata (T2), além disso, os valores de Gleason possuíam uma incidência bastante heterogênea, divergindo assim, com os dados encontrados na presente pesquisa (CAMBRUZZI E, et al., 2010; BARONI RH, et al., 2009; SILVA TA, et al., 2014).

Conforme a sintomatologia foi observado que a maioria se apresentava assintomático durante o tratamento. Porém, vale ressaltar que dor óssea, nictúria, disúria, polaciúria, incontinência urinária, disfunção erétil, ginecomastia, dores musculares e nas articulações também foram relatadas. A maioria dos sintomas estão associados ao tratamento, pois o uso da hormonioterapia pode causar efeitos colaterais como, impotência, osteoporose e sensibilidade e/ou crescimento nas mamas (PORTUGAL, 2007).

No entanto, com relação os sintomas próprios do câncer de próstata relatados, como nictúria, polaciúria, dor óssea, incontinência urinária e dor nos membros inferiores corroboram com a literatura, porém houve diferenças na frequência relativa de cada sintoma (GONÇALVES IR, et al., 2008).

O perfil dos pacientes acometidos com câncer de próstata na região pesquisada é na maioria idosos, analfabetos, casados, agricultores e procedentes de áreas rurais. Esse perfil no geral corrobora com o estudo de base populacional de Lima AP, et al. (2018), ressaltando o apoio familiar de pacientes casados que são um dos principais estímulos para que os pacientes realizem exames preventivos com frequência, deixando em evidência que há um forte estigma social de preconceitos e desinformação sobre a realização do exame para o diagnóstico precoce.

O diagnóstico tardio foi um dos dados clínicos observados com predominância, visto uma resistência dos homens em cuidar da saúde devido a vergonha/exposição do corpo, ao medo da descoberta de doenças graves em consistência com o modelo hegemônico da masculinidade. Isso reflete no alerta para intensificar estratégias que comportem esse público e que possa desmistificar esses fatores (SANTOS ASF, et al., 2020). Além disso, a maioria era ex-fumantes, apresentavam uma história pregressa de etilismo e tinham um contato frequente com agrotóxicos no trabalho. Todos esses fatores são os maiores desafios para a Atenção Primária em Saúde para criar medidas de prevenção e promoção integrando a Política Nacional de Atenção Integral à Saúde do Homem para incentivar estilos de vida saudáveis (OLIVEIRA PSD, et a., 2019; SANTOS ASF, et al., 2020).

Diante do panorama do câncer de próstata nesse estudo as variáveis de maior importância para a observação do perfil clínico desses indivíduos se reflete nas características sociais consolidando como variáveis preditivas da localidade. Essas características corroboram com os estudos de coorte observado por Braga SFM (2016), que demonstra a sobrevida e fatores prognósticos dos indivíduos colocando o perfil de morbidade como um problema de saúde pública. O estudo apresenta limitações no que se refere à coleta de dados, pois vários prontuários consultados havia a falta de informações quanto ao estadiamento do paciente. Dessa forma, tais dados incompletos dificultaram a análise desta variável neste estudo.

\section{CONCLUSÃO}

Considerando a heterogenia da realidade encontrada nas diversas regiões do país e seu impacto no processo saúde-doença, é de suma importância o conhecimento das especificidades da população atendida no centro de referência em oncologia no município, contribuindo assim na melhoria do processo do cuidar, na perspectiva de uma atenção holística e específica. Dessa forma, o conhecimento da realidade epidemiológica, sociocultural e clínica dos homens acometidos com câncer de próstata na região podem contribuir na elaboração de políticas públicas direcionadas para essa população e assim adotar de medidas de deteç̧ão, prevenção, promoção e rastreamento mais eficazes, melhorando o prognóstico da doença.

\section{REFERÊNCIAS}

1. ABAZA R, et al. Prognostic value of DNA ploidy, bcl-s and p53 in localized prostate adenocarcinoma incidentally discovered at transurethral prostatectomy. J Urol, 2006; 176(5) 2701-5.

REAEnf/EJNC | Vol. 8 | e5855 | DOI: https://doi.org/10.25248/REAenf.e5855.2021 Página 8 de 9 
2. ABDOLLAH F, et al. The effect of marital status on stage and survival of prostate cancer patients treated with radical prostatectomy: a population-based study. Cancer Causes Control. 2011; 22(8) 1085-95.

3. LIMA AP, et al. Prevalência e fatores associados à realização de exames de câncer de próstata em idosos: estudo de base populacional. Rev. Bras. Geriatr. Gerontol., Rio de Janeiro, 2018; 21(1): 55-61.

4. BARONI RH, et al. Ressonância magnética da próstata: uma visão geral para o radiologista. Radiol Bras, $2009 ; 4$ 2(3) 185-192.

5. BRAGA SFM. Câncer da próstata: sobrevida, fatores associados ao risco de óbito e tendência de mortalidade no brasil. Tese (Doutorado em Saúde Pública) - Universidade Federal de Minas Gerais, Belo Horizonte, $2016 ; 113$ p.

6. BRASIL. Programa nacional de controle do câncer da próstata: documento de consenso. Rio de Janeiro: 2002; 24p.

7. CAMBRUZZI E, et al. Relação entre escore de Gleason e fatores eprognósticos no adenocarcinoma acinar de próstata. J Bras Patol Med Lab, 2010; 46(1) 61-68.

8. CZORNY RCN, et al. Fatores de risco para o câncer de próstata: população de uma unidade básica de saúde. Cogitare Enferm. 2017; (22) 4: e51823.

9. DINI LI, KOFF WJ. Perfil do câncer de próstata no hospital de clínicas de Porto Alegre. Rev. Assoc. Med. Bras, 2006; 52(1) 28-31.

10. GONÇALVES IR, et al. Caracterização epidemiológica e demográfica de homens com câncer de próstata. Ciência e Saúde Coletiva, 2008; 13(4) 1337-1342.

11. GRONBERG H. Prostate cancer epidemiology. Lancet, 2003; 361: 859-64.

12. HAAS GP et al. The worldwide epidemiology of prostate cancer: Perspectives from autopsy studies. Can J Urol 2008; 15(1) 3866-3871.

13. INCA. Instituto Nacional do Câncer. Estimativa de câncer no Brasil: Incidência de câncer no Brasil/ Instituto Nacional de Câncer José Alencar Gomes da Silva - Rio de Janeiro, 2020.

14. KRAVDAL A. Cancer survival model that takes sociodemographic variations in "normal" mortality into account: comparison with other models. J Epidemiol Community Health, 2002; 56(4) 309-18.

15. KUMAR V, et al. CY1P1 polymorphism and organoclorine pesticides leves in the etioloy of prostate cancer. Chemosphere 2010; (81) 464-468.

16. MOSCHETA MS, SANTOS MA. Grupos de apoio para homens com câncer de próstata: revisão integrativa da literatura. Ciênc. Saúde Coletiva, 2012; 17(5) 1225-1233.

17. NASSIF AE, et al. Perfil epidemiológico e fatores prognósticos no tratamento cirúrgico do adenocarcinoma de próstata clinicamente localizado. Rev. Col. Bras. Cir. 2009; 36(4) 327-31.

18. OLIVEIRA P S D et al. Câncer de próstata: conhecimentos e interferências na promoção e prevenção da doença. Enfermería Global № 54 Abril 2019. Pag 262-273.

19. PAIVA EP, et al. Conhecimentos, atitudes e práticas acerca da detecção do câncer de próstata. Acta Paul Enferm, 2010; 23(1) 88-89.

20. PORTUGAL. Associação Portuguesa de Urologia. Sociedade Portuguesa de Oncologia. Cancro da Próstata: tudo o que você precisa saber. Sanof Oncology. Bloom up, pt.cab:12.09.2007.

21. SANTOS ASF, et al., 2020. Internações por câncer de próstata em uma regional de saúde do estado de Pernambuco e as relações com as possibilidades de prevenção na atenção primária. J Manag Prim Health Care, 2020;12:e32.

22. SIDDIQUI AS, et al. Impact of familial and hereditary prostate cancer on cancer specific survival after radical retropubic prostatectomy. J Urol, 2006; 176(3) 1118-21.

23. SILVA TA, et al. Caracterização do câncer de próstata entre pacientes pertencentes à raça negra. Ciencia er Praxis, $2014 ; 7(13)$.

24. ZACCHI SR, et al. Associação de variáveis sociodemográficas e clínicas com o estadiamento inicial em homens com câncer de próstata. Cad Saúde Colt, 2014; 22(1):93-100. 